Article

\title{
Pyrolyzing Renewable Sugar and Taurine on the Surface of Multi-Walled Carbon Nanotubes as Heterogeneous Catalysts for Hydroxymethylfurfural Production
}

\author{
Huiping $\mathrm{Ji}^{1}{ }^{1}$, Jie $\mathrm{Fu}^{2}{ }^{2}$ and Tianfu Wang ${ }^{3, *}$ \\ 1 University of Chinese Academy of Sciences, Beijing 100049, China; jihuiping15@mails.ucas.ac.cn \\ 2 Key Laboratory of Biomass Chemical Engineering of Ministry of Education, College of Chemical and \\ Biological Engineering, Zhejiang University, Hangzhou 310027, China; jiefu@zju.edu.cn \\ 3 Xinjiang Technical Institute of Physics \& Chemistry, Chinese Academy of Sciences, Urumqi 830011, China \\ * Correspondence: tianfuwang@ms.xjb.ac.cn
}

Received: 12 August 2018; Accepted: 21 September 2018; Published: 5 November 2018

\begin{abstract}
Conversion of biorenewable feedstocks into transportation fuels or chemicals likely necessitates the development of novel heterogeneous catalysts with good hydrothermal stability, due to the nature of highly oxygenated biomass compounds and the prevalence of water as a processing solvent. The use of carbon-based materials, derived from sugars as catalyst precursors, can achieve hydrothermal stability while simultaneously realizing the goal of sustainability. In this work, the simultaneous pyrolysis of glucose and taurine in the presence of multi-walled carbon nanotubes (MWCNTs), to obtain versatile solid acids, has been demonstrated. Structural and textural properties of the catalysts have been characterized by TEM, TGA, and XPS. Additionally, solid state nuclear magnetic resonance (ssNMR) spectroscopy has been exploited to elucidate the chemical nature of carbon species deposited on the surface of MWCNTs. $\mathrm{Al}(\mathrm{OTf})_{3}$, a model Lewis acidic metal salt, has been successfully supported on sulfonic groups tethered to MWCNTs. This catalyst has been tested for $\mathrm{C}_{6}$ sugar dehydration for the production of $\mathrm{HMF}$ in a tetrahydrofuran (THF)/water solvent system with good recyclability.
\end{abstract}

Keywords: carbon nanotubes; carbohydrates; HMF; Lewis acids; NMR

\section{Introduction}

Due to the rapidly growing world energy and materials consumption, and diminishing petroleum reserves, economical and environmental concerns have risen, urging the utilization of renewable sources of carbon for liquid transportation fuels and chemicals. Biomass is uniquely situated to provide an alternative to petroleum-sourced carbon because of its abundance and sustainability [1]. However, in contrast to traditional feedstocks, molecules derived from the carbohydrate fractions of biomass consist of highly oxygenated compounds. This necessitates the removal of functionality before biomass can replace the highly reduced carbon platform molecules typical of the modern fuel and chemical industry [2]. Therefore, processing of these materials will greatly differ from the functionality-adding processes that have been well-researched and employed in the petrochemical industry. The need to adapt to these new feedstocks and processing conditions is particularly challenging for the field of heterogeneous catalysis [3]. It is important that the heterogeneous catalysis community performs research with common biorenewable compounds, in order to gain understanding and subsequent ability to control the active site environment, as well as develop catalysts with hydrothermal stability [3]. 
Common catalysts employed in the petroleum industry, such as zeolites, have been shown to be unstable with exposure to hot water. Stability must be considered for biomass upgrading [4]. Consequently, hydrothermal stability concerns have promoted recent research into carbon-based supports for biorenewable applications [5]. One particular carbon catalyst support receiving a significant amount of attention in recent years is that of carbon nanotubes. Multi-walled carbon nanotubes (MWCNTs) are a hydrothermally stable material, and they offer other reasons that make them an interesting material as well. For instance, they offer uniform and tunable textural properties, such as porosity, that can be easily manipulated. This allows for greater control over the catalyst active site than is available when using conventional carbon-based materials, such as activated carbon. MWCNTs also have a relatively high surface area and relatively less microporosity, making them less likely than conventional carbon-based materials to encounter mass transport limitations when they are used as catalyst supports [6].

Previously, a simple method to produced functionalized carbon rich materials, from glucose and a wide number of molecules containing a primary amine, was demonstrated, including $\left(\mathrm{CH}_{2}\right)_{2}-\mathrm{SO}_{3} \mathrm{H}$ groups, which would be ideal to coordinate metal cations, and are strong Brønsted acids [7]. The hydrothermal stability of sulfonated carbon materials has been called into question in the past, and it has been shown that $\mathrm{SO}_{3} \mathrm{H}$ groups directly bonded to aromatic carbons are substantially less stable than $\mathrm{SO}_{3} \mathrm{H}$ groups bonded to alkyl $\mathrm{CH}_{2}$ [8]. In an earlier study, it was found that glucose and taurine can be pyrolyzed together to form alkyl-linked sulfonic acids for various acid-catalyzed reactions, such as dehydration and esterification [9], and this class of materials has been thoroughly characterized by NMR [10]. However, solid acids produced from this strategy suffer from their intrinsically low surface area, typically $\sim 1 \mathrm{~m}^{2} / \mathrm{g}$, limiting their potential applications in catalysis. In order to solve this problem, we reasoned that, in the presence of a porous support such as MWCNT, co-pyrolysis of glucose and taurine could form, ideally, a thin layer of carbonaceous material containing alkyl-linked sulfonic acid groups. The resulting high surface area solid acids could be applicable in a range of acid-catalyzed reactions.

Recently, increasing interest has been attracted for the development of biorenewable plastics derived from the carbohydrate portion of lignocellulosic biomass [11]. Among all the proposed platform chemicals that can be converted to monomers for subsequent production of polymers, 5-hydroxymethylfurfural (HMF) has been identified as a promising platform chemical candidate for the production of polyethylene furanics (PEF) to replace petroleum-based polyethylene terephthalate (PET). In the past decade, various catalysts, such as homogeneous sulfonic acid [11], heteropolyacids (HPAs) [12], heterogeneous metal-oxide-based catalysts, like tin-beta zeolites [13] and $\mathrm{Nb}_{2} \mathrm{O}_{5}$ [14], have all been reported to catalyze the dehydration reaction of $\mathrm{C}_{6}$ monosaccharide to HMF. In addition, numerous green solvent and catalytic systems have been demonstrated to show high glucose-HMF conversion activity and good yields of HMF have been reported, inspiring the development of new class of catalysts [15-17]. Very recently, a class of metal salts, such as $\mathrm{AlCl}_{3}$ [18], $\mathrm{SnCl}_{4}$ [19], $\mathrm{GeCl}_{4}$ [20], and $\mathrm{YbCl}_{3}$ [21], dissolved in either aqueous or ionic liquid media, have been investigated as extremely effective catalysts for HMF production. The unique catalytic performance of these metal salt catalytic systems has been attributed to the combination of the Lewis and Brønsted nature possessed by metal cations and, hence, facile interaction with sugar molecules to promote HMF synthesis [18]. Although promising, recycling and separation of the homogeneous metal salts may raise concerns on the practicality of such systems. In order to solve this dilemma, it could be advantageous to immobilize these metal salts on a solid support.

In this study, we co-pyrolyzed glucose and taurine to disperse a carbonaceous layer onto MWCNTs, and tested the reaction kinetics, hydrothermal stability, and $\mathrm{SO}_{3} \mathrm{H}$ bonding environment of this catalyst. Co-pyrolysis of glucose and taurine with MWCNT, under controlled experimental conditions $\left(200^{\circ} \mathrm{C}\right.$ and $\left.250^{\circ} \mathrm{C}\right)$, resulted in a hybrid material in which sulfonic groups were present on the surface of MWCNT. Solid-state NMR was used to determine the chemical structure of the hybrid material and the bonding nature of the sulfonic group using ${ }^{13} \mathrm{C}$-labeled glucose and ${ }^{13} \mathrm{C}$ - or 
${ }^{15} \mathrm{~N}$-labeled taurine. The electrostatic interaction between sulfonate and the aluminum cation was exploited for immobilization of the model Lewis acid catalyst, $\mathrm{Al}(\mathrm{OTf})_{3}$. Lewis acid-immobilized MWCNT catalysts were subsequently tested for their activities in glucose and fructose dehydration reactions, as shown in Figure 1. In addition, catalyst recyclability studies using fructose were carried out, and Al leaching tests with ICP analysis were performed after each run, to probe catalyst stability under reaction conditions.

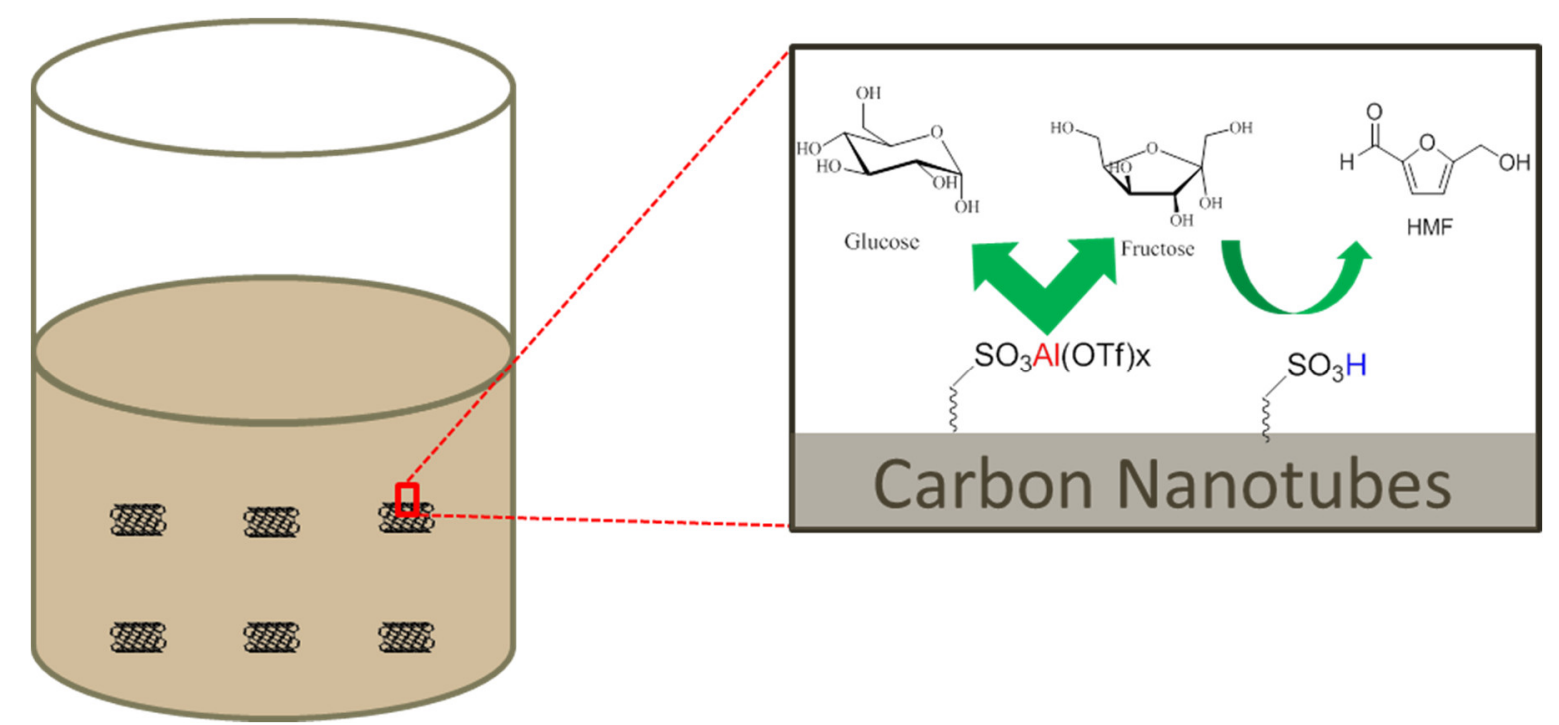

Figure 1. Solid Lewis/Brønsted acid catalysts for hydroxymethylfurfural (HMF) production from $\mathrm{C}_{6}$ sugars.

\section{Results and Discussion}

\subsection{Catalyst Characterizations}

Sulfonic acid groups were introduced to the surface of MWCNT by co-pyrolyzing glucose and taurine. Before pyrolysis, the parent MWCNT has a BET surface area of $304 \mathrm{~m}^{2} / \mathrm{g}$ and pore volume of $1.13 \mathrm{~cm}^{3} / \mathrm{g}$, which were reduced to $118 \mathrm{~m}^{2} / \mathrm{g}$ and $0.33 \mathrm{~cm}^{3} / \mathrm{g}$, respectively. This change in porosity properties provides indirect evidence of the successful deposition of functional groups on the surface of MWCNT. TEM and EDS imaging studies have provided further confirmation of the successful synthesis of sulfonate-tethered MWCNT catalysts. As shown in Figure 2, the representative solid Lewis acid catalysts (after the immobilization of $\mathrm{Al}(\mathrm{OTf})_{3}$ ) revealed the typical morphology of a multi-walled carbon nanotube (see Figure 2a). EDS mapping revealed a homogeneous distribution of sulfur, indicating that the sulfonic groups are impregnated throughout the MWCNT surface. Further EDS data is summarized in Figure 3 for sulfur, carbon, oxygen, and aluminum. By comparing the EDS data, it can be concluded that both $\mathrm{Al}$ and $\mathrm{S}$ are well-dispersed throughout the MWCNT surface, proving the effectiveness of the pyrolysis and immobilization as catalyst preparation methods. Additionally, shown in Figure $2 c, d$ are the images of the Lewis acid catalyst after being subjected to the reaction condition of $160^{\circ} \mathrm{C}$ in THF/water for $3 \mathrm{~h}$, referred to as the "spent" catalyst. Although ICP analysis of the supernatant would be a more definitive method to quantify the stability of the immobilized $\mathrm{Al}$, the visual TEM/EDS images demonstrate a similar morphology and S distribution for both the intact and spent Lewis acid catalysts. This issue is discussed further below. 

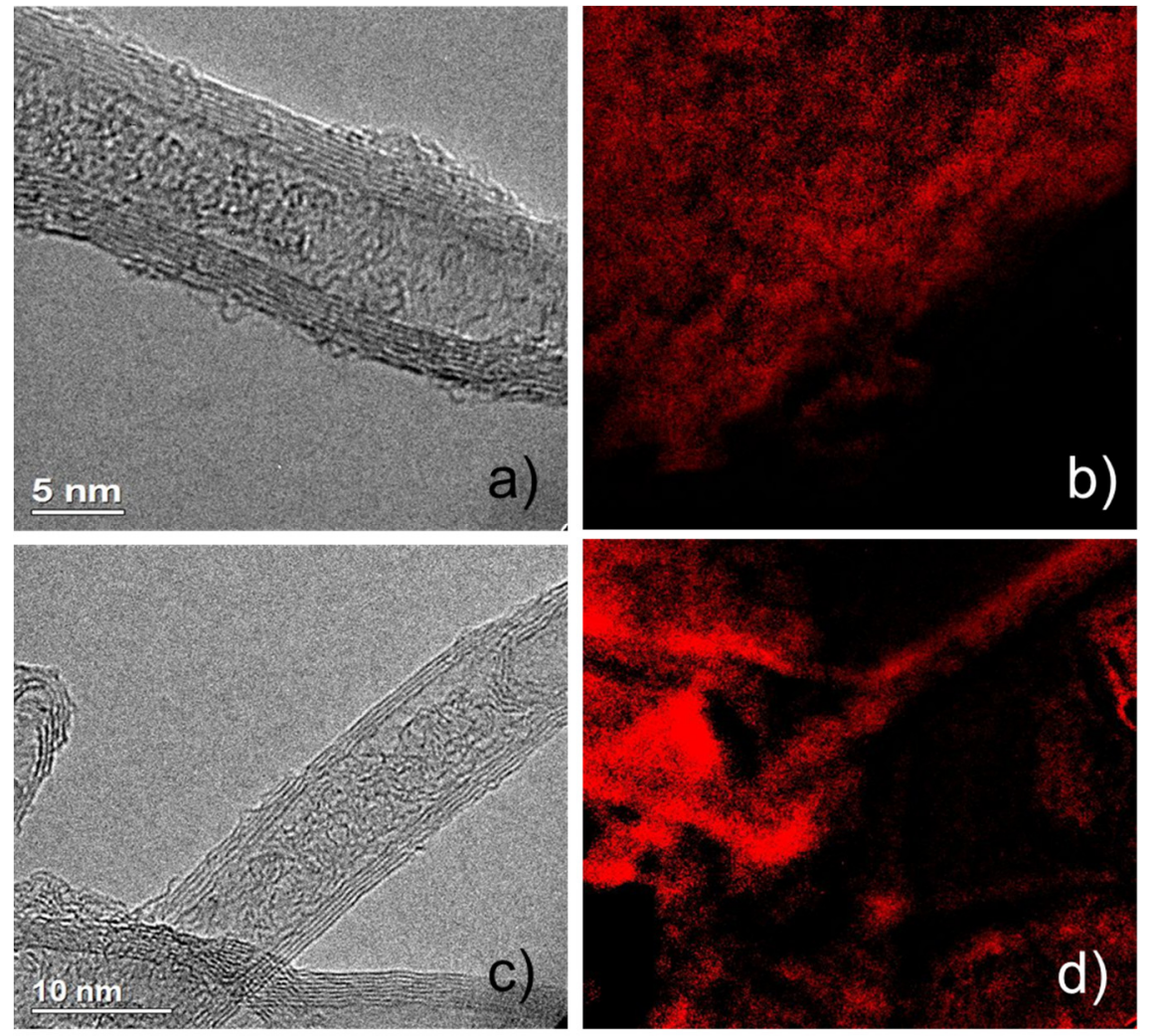

Figure 2. TEM and EDS images of (a) LA-MWCNT (Lewis acid-multi-wall carbon nanotube); (b) S mapping of LA-MWCNT; (c) spent LA-MWCNT; and (d) S mapping of spent LA-MWCNT.

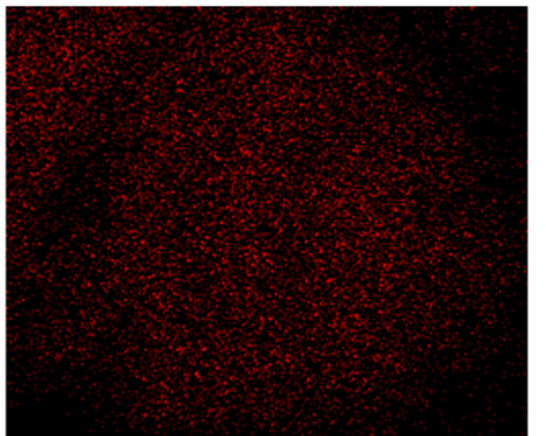

CKa1_2

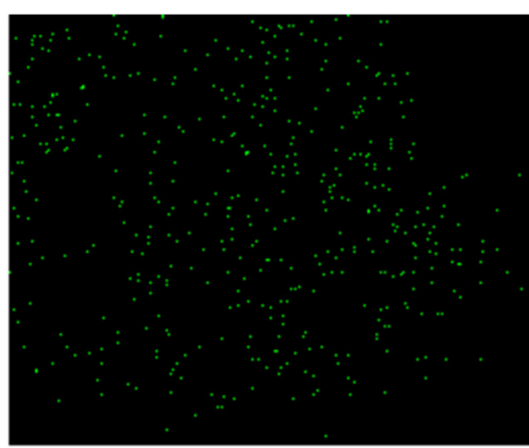

Al Ka1

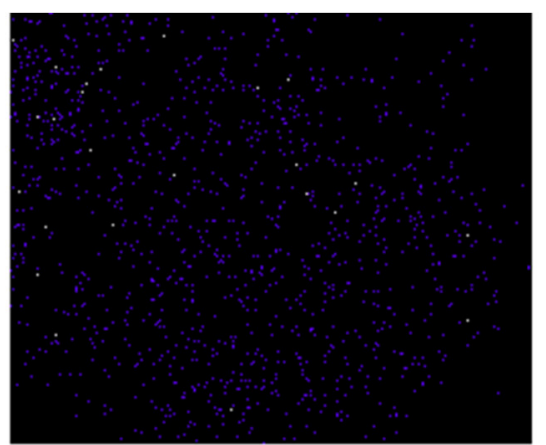

O Ka1

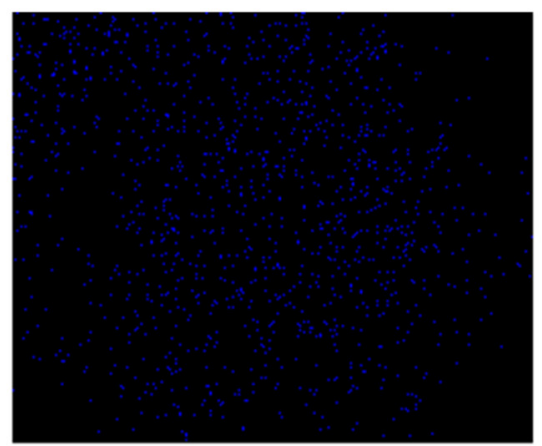

SKa1

Figure 3. EDS elemental mapping of LA-MWCNT. 


\subsection{Solid-State NMR}

The aromaticity of each material is quantified by measuring the area under the aromatic peak in the ${ }^{13} \mathrm{C}$ spectrum as a percentage of the total area under the spectrum. Since glucose makes up $30 \mathrm{~mol} \%$ of the carbon in the MWCNT-containing samples, and only $1 \%$ of the remaining carbon is ${ }^{13} \mathrm{C}$, the intensity in the DP/MAS spectra shown in Figure 4 may be considered to be entirely from the ${ }^{13} \mathrm{C}$ nuclei that originated in the glucose. By this method, the fate of $69 \%$ of the glucose carbon in GT250/1-GC is to become aromatic, while 72\% of the glucose carbon in GT250/10-CNT-GC becomes aromatic. This indicates that the presence of the MWCNT during the pyrolysis reaction at $250{ }^{\circ} \mathrm{C}$ does not significantly change the aromatic fraction of the pyrolysis product.

Figure 4 also shows DP/MAS experiments with dipolar dephasing, a method which suppresses signals from ${ }^{13} \mathrm{C}$ species with strong dipolar couplings to ${ }^{1} \mathrm{H}$. Therefore, the spectra with dipolar dephasing selectively show signal from nonprotonated and very mobile ${ }^{13} \mathrm{C}$ species. Sample GT250/10-CNT-GC loses less signal than GT250/1-GC in the dipolar dephasing experiments (Figure 4), indicating that fewer of the ${ }^{13} \mathrm{C}$ nuclei contributed to the material by glucose end up protonated when the reaction is carried out with MWCNT. To further probe this reduced protonation, we applied DP-based ${ }^{13} \mathrm{C}$ [22] REDOR experiments to the glucose-labeled samples. The aromatic ${ }^{13} \mathrm{C}$ nuclei in GT250/10-CNT-GC are dephased more slowly than those in GT250/1-GC. Dephasing in a REDOR experiment occurs slower when ${ }^{1} \mathrm{H}$ nuclei are farther away from the ${ }^{13} \mathrm{C}$. The REDOR results indicate that the aromatic domains are larger when MWCNTs are present, than in the $250{ }^{\circ} \mathrm{C}$ pyrolysis product alone, even though the aromatic fraction is similar.

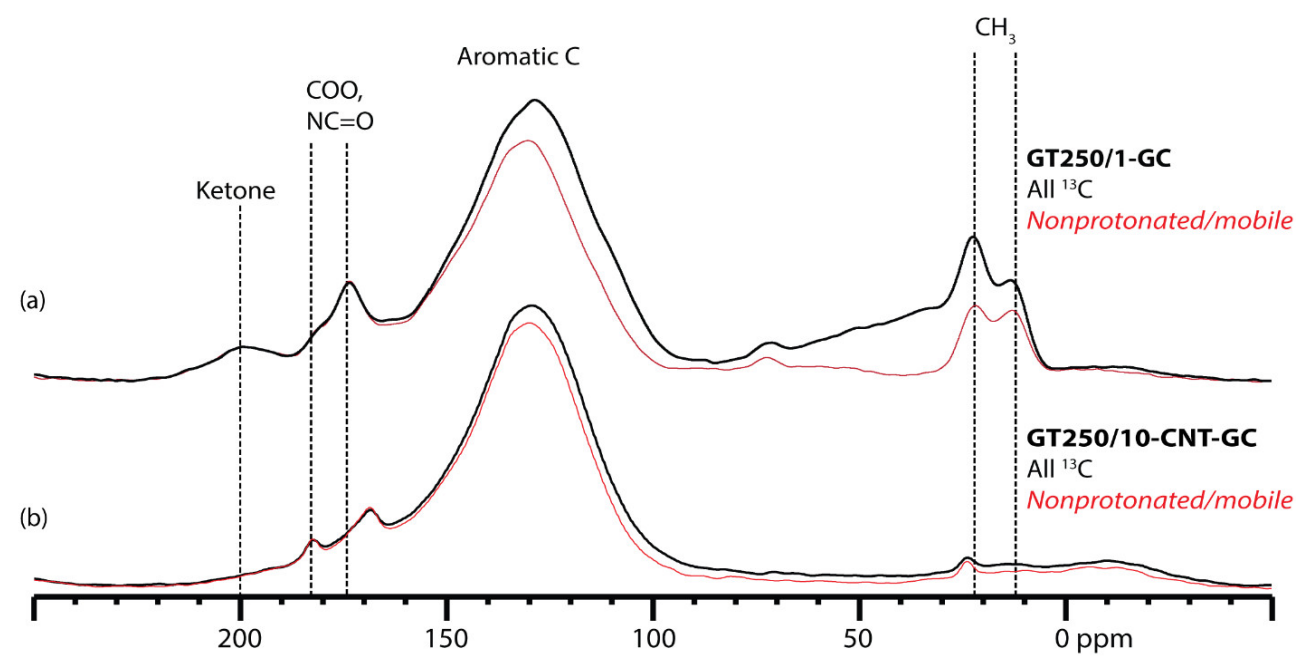

Figure 4. ${ }^{13} \mathrm{C}$ NMR spectra of ${ }^{13} \mathrm{C}$-glucose-labeled glucose-taurine materials. Synthesis details in Table 1. (a) Pyrolyzed product prepared without MWCNTs. Thick black trace: DP spectrum of all ${ }^{13} \mathrm{C}$ species, $69 \%$ of which are aromatic. Thin red trace: nonprotonated or highly mobile ${ }^{13} \mathrm{C}$ species, obtained by dipolar dephasing; (b) Pyrolyzed product prepared with MWCNTs. Thick black trace: multiCP spectrum of all ${ }^{13} \mathrm{C}$ species, $72 \%$ of which are aromatic. Thin red trace: nonprotonated or highly mobile ${ }^{13} \mathrm{C}$ species, obtained by dipolar dephasing.

In the $4 \mathrm{~mm}$ probehead experiments on samples containing ${ }^{13} \mathrm{C}$-labeled taurine, no indication was found of methylene groups, which would be characteristic of an alkyl link between the taurine sulfonate and the bulk of the pyrolyzed product. However, as described in the experimental section, incomplete ${ }^{1} \mathrm{H}$ decoupling, due to conductivity-induced power loss, can cause alkyl ${ }^{13} \mathrm{C}$ to be underrepresented. For that reason, NMR experiments on the ${ }^{13} \mathrm{C}$-taurine samples were repeated using the $2.5 \mathrm{~mm}$ probehead to ensure that any existing methylene groups were observed (Figure 5). In these experiments, fewer scans were needed to compile a spectrum with a similar level of signal-to-noise (also due to the lower loss of power in the $2.5 \mathrm{~mm}$ probehead). The ${ }^{1} \mathrm{H}$ decoupling achieved in the $2.5 \mathrm{~mm}$ probehead 
was over $71 \mathrm{kHz}$, as determined by the $90^{\circ}{ }^{1} \mathrm{H}$ pulse time of $3.5 \mu \mathrm{s}$. For the $2.5 \mathrm{~mm}$ probehead spectra, the quantitative multiCP experiment was used instead of DP, since that experiment is much faster. The multiCP experiment was not used in the $4 \mathrm{~mm}$ probehead because it relies heavily on efficient ${ }^{1} \mathrm{H}$ pulses, which were not achieved in the $4 \mathrm{~mm}$ probehead, due to power loss.

In the GT250/10-CNT-TC quantitative spectrum from the $2.5 \mathrm{~mm}$ probehead (Figure $5 \mathrm{a}$ ), as in the $4 \mathrm{~mm}$ probehead, no characteristic peaks from the alkyl moiety contributed by taurine are seen. Since a failure to sufficiently decouple the methylene ${ }^{13} \mathrm{C}$ nuclei from their ${ }^{1} \mathrm{H}$ bonding partners is ruled out in the 2.5-mm probehead, we can say that the alkyl moiety is, in fact, missing from that material, and no alkyl-linked sulfonate is expected to exist.

Two variations of the ${ }^{13} \mathrm{C}$-taurine-labeled material with MWCNT were prepared to examine the effect on reaction temperature and the rate of temperature increase on the retention of the taurine methylene moiety in the post-pyrolysis structure. Both of these samples were analyzed using multiCP with and without recoupled dipolar dephasing in the $2.5 \mathrm{~mm}$ probehead. The spectra are shown for comparison with that of GT250/10-CNT-TC (Figure 5). The sample that was pyrolyzed at $250{ }^{\circ} \mathrm{C}$, but was brought up to that temperature more slowly (GT250/1-CNT-TC), shows similar relative intensities in the aromatic and aliphatic regions as GT250/10-CNT-TC (Figure 5). The ${ }^{13} \mathrm{C}$ from taurine is distributed between the aromatic framework, and any remaining alkyl groups in about the same way as in the faster-ramped sample. However, the slower-ramped sample shows a larger loss from dipolar dephasing in the aromatic regions. This indicates that more of the aromatic ${ }^{13} \mathrm{Cs}$ in the slower-ramped sample are protonated. A larger fraction of protonated carbon indicates that the taurine ${ }^{13} \mathrm{C}$ nuclei become incorporated into smaller aromatic domains in the slower-ramped sample.

Compared to the products of pyrolysis at $250{ }^{\circ} \mathrm{C}$, the ${ }^{13} \mathrm{C}$-taurine sample pyrolyzed at $200{ }^{\circ} \mathrm{C}$ (GT200/1-CNT-TC) shows less intensity in the aromatic region and more intensity in the region associated with methylene groups. This demonstrates that a lower reaction temperature can increase the retention of the alkyl moiety of taurine in the final product. The intensity in the alkyl region is in the range expected for $\mathrm{N}-\mathrm{CH}_{2}$ and $\mathrm{S}-\mathrm{CH}_{2}$ bonding, which is evidence for the retention of the entire $\mathrm{N}\left(\mathrm{CH}_{2}\right)_{2} \mathrm{SO}_{3}$ sequence. Since the intensity in this region is composed of overlapping peaks, the determination of the exact bonding environment of these methylene groups would require some modeling of potential structures, probably in combination with 2D NMR experiments. The predicted ${ }^{13} \mathrm{C}$ and ${ }^{15} \mathrm{~N}$ NMR spectra based on those structures could then be compared to the experimental results for the ${ }^{13} \mathrm{C}$-labeled and ${ }^{15} \mathrm{~N}$-labeled samples.

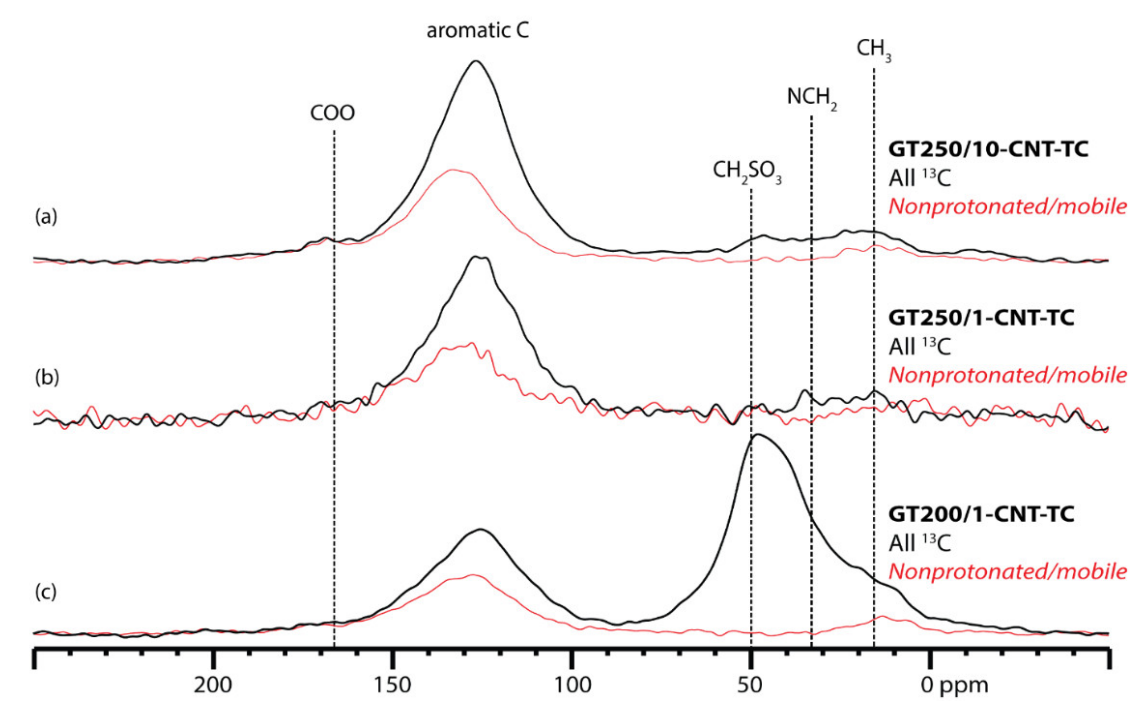

Figure 5. MultiCP ${ }^{13} \mathrm{C}$ NMR spectra of ${ }^{13} \mathrm{C}$-taurine-labeled glucose-taurine pyrolysis products, all prepared with MWCNTs. Synthesis details in Table 1. Thick black traces: multiCP spectrum of all ${ }^{13} \mathrm{C}$ species. Thin red traces: nonprotonated or highly mobile ${ }^{13} \mathrm{C}$ species, obtained by dipolar dephasing. Fraction of aromatic species (a) $80 \%$; (b) $76 \%$; and (c) $24 \%$. 


\subsection{Reaction Testing}

Initial reaction testing of each catalyst was performed in a binary solvent consisting of THF and DMSO. This is a well-known reaction solvent to minimize side reactions. As shown in Supplemental Figure 2, the glucose/fructose conversion kinetics are highly dependent on the catalysts used. Very little glucose conversion was observed in the absence of catalysts added. All of the added catalyst combinations can promote glucose conversion to HMF, as can be seen in the plot. The greatest improvement was shown by the solid Lewis and Brønsted acid catalyst, and the solid Lewis acid catalyst, both of which were more effective than the solid Brønsted acid catalyst. This is in agreement with our previous work. However, for all of the glucose reactions, low selectivity, ranging from 0 to $20 \mathrm{~mol} \%$, was obtained. This can be ascribed to undesired side reactions on the surface of MWCNTs, and likely be associated with metal cation catalysis to induce the formation of humins and other side products. Since solid Lewis acid alone was found to be quite active for the catalysis, a combination of Lewis and Brønsted acid was chosen to be used for later conversions. Unlike for glucose, catalytic performance of the solid catalysts for fructose was improved significantly by adding Brønsted acid functionality. The conversion kinetics showed that at a reaction temperature lower than $120^{\circ} \mathrm{C}$, fructose conversion was completed within the first $3 \mathrm{~h}$ with selectivity greater than $85 \mathrm{~mol} \%$.

Based on the initial reactivity/selectivity trends, we chose to use the THF/water binary solvent system and a combination of solid Lewis and Brønsted acid catalysts for further reaction testing. Although DMSO can generally enhance sugar dehydration selectivity by minimizing side reactions, its practical implementation can be problematic, due to its high boiling point and cost. Figure 6 summarizes the reaction result of fructose conversion using reaction media of THF/water and reaction temperature of $160^{\circ} \mathrm{C}$. Conversion rate using fructose as feedstock was faster than glucose, as was observed in THF/DMSO solvent. In addition, selectivity of HMF from fructose was about $60 \mathrm{~mol} \%$, while less than $10 \mathrm{~mol} \%$ selectivity from glucose was achieved.
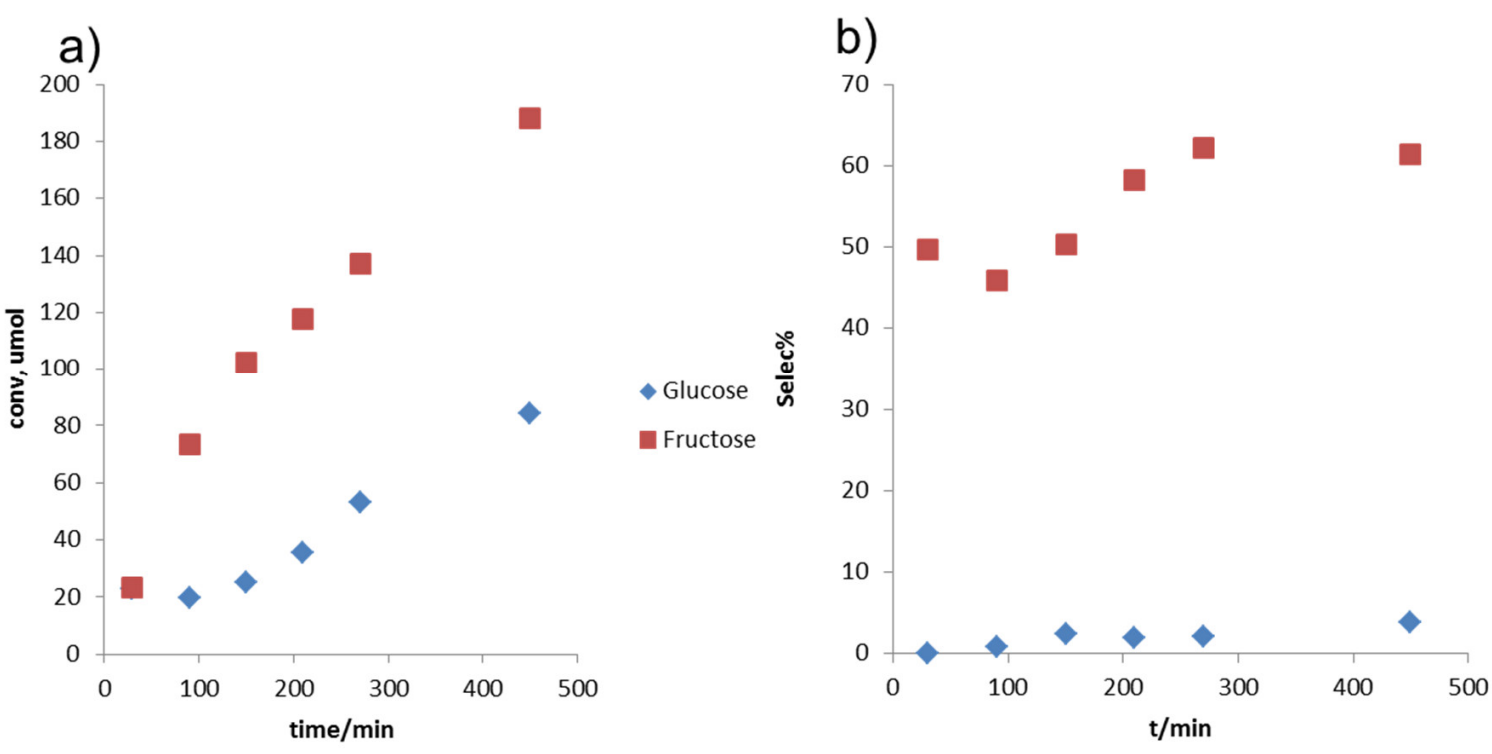

Figure 6. Reaction results of glucose/fructose conversion in THF/water solvent. (a) Conversions; (b) selectivities. Reaction conditions: solvent of THF/water 9:1 $(v / v)$, reaction temperature of $160{ }^{\circ} \mathrm{C}$, $2 \mathrm{wt} \%$ glucose/fructose, $5 \mathrm{mg}$ each of Lewis and Brønsted catalyst/g of reaction media.

Catalyst stability and recyclability under reaction conditions are of great importance in industrial applications. Regarding this aspect, the recyclability of the solid catalysts for the fructose reaction was tested. As shown in Figure 7 and described above, three consecutive reactions were run with fructose as feedstock following the same procedure. The reaction kinetic profile showed a relatively similar reaction rate between the three runs, with little loss of catalyst activity. Selectivity for HMF was also 
kept around $60 \mathrm{~mol} \%$, which was slightly lower than THF/DMSO solvent system. The supernatant after each reaction was also analyzed for possible Al leaching. The calculated Al leaching was found to be $3.3 \%, 2.2 \%$, and $1.8 \%$, respectively.
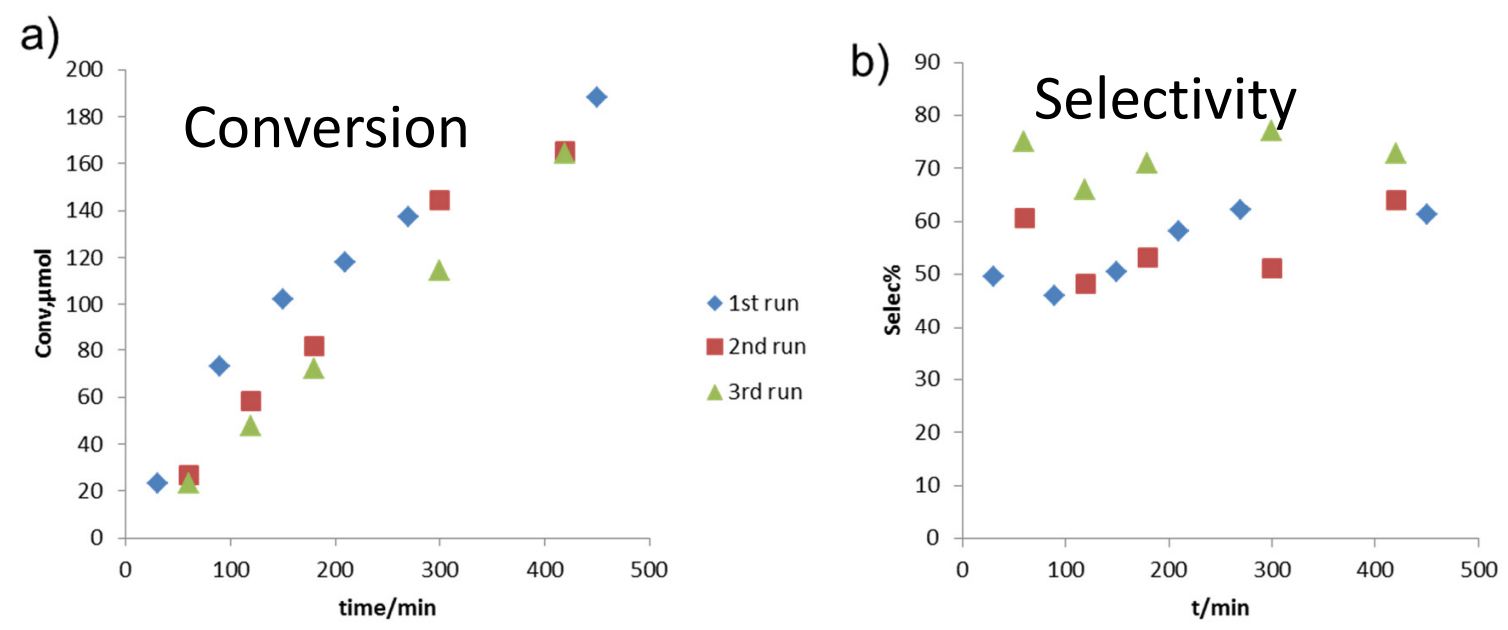

Figure 7. Recyclability study with fructose as feedstock. Reaction conditions: solvent of THF/water 9:1 $(v / v)$, reaction temperature of $160{ }^{\circ} \mathrm{C}, 2 \mathrm{wt} \%$ fructose, $5 \mathrm{mg}$ each of Lewis and Brønsted catalyst/g of reaction media.

\section{Experimental}

\subsection{Catalyst Synthesis}

Before the sulfonate-tethering reaction, the MWCNTs (Timenano, Chengdu, China) were ultrasonicated using a Branson Digital Sonifier ${ }^{\circledR}$ (Emerson, St. Louis, MO, USA) for 15 min with $90 \%$ power output, to remove amorphous carbon and introduce oxygenated groups to the surface. To synthesize sulfonate-tethered MWCNTs, an aqueous solution of $0.71 \mathrm{M}$ glucose (Fisher, Hampton, $\mathrm{NH}$, USA), $0.355 \mathrm{M}$ taurine (Sigma-Aldrich, St. Louis, MO, USA), and $0.355 \mathrm{M} \mathrm{NaOH}$ (Fisher) was mixed with MWCNT (combined weight glucose and taurine accounts for $15 \mathrm{wt} \%$ carbon content relative to the weight of MWCNT). The mixture was then sonicated for $5 \mathrm{~min}$, followed by heating to $120^{\circ} \mathrm{C}$ with ramp rate of $1{ }^{\circ} \mathrm{C} / \mathrm{min}$ or $10^{\circ} \mathrm{C} / \mathrm{min}$, and held for $3 \mathrm{~h}$ until dry. The pyrolysis was done at $200{ }^{\circ} \mathrm{C}$ or $250^{\circ} \mathrm{C}$ under $200 \mathrm{~mL} / \mathrm{min}$ flowing $\mathrm{N}_{2}$ protection, with a temperature ramping rate of either $1{ }^{\circ} \mathrm{C} / \mathrm{min}$ or $10^{\circ} \mathrm{C} / \mathrm{min}$, and held at the final temperature for additional $10 \mathrm{~h}$. The same protocol was repeated again to increase the number of functional groups introduced. Table 1 summarizes the synthesis conditions for different pyrolysis products.

To make the solid Lewis acid (LA)-MWCNT, $0.8 \mathrm{~g}$ of the sulfonate-tethered MWCNTs, synthesized by the method described above, were first immersed in $1 \mathrm{M} \mathrm{NaCl}$ solution and stirred for $24 \mathrm{~h}$, followed by contacting with $\mathrm{Al}(\mathrm{OTf})_{3}$ (Sigma-Aldrich, $1.0 \mathrm{~g}$ ) in ethanol at $80^{\circ} \mathrm{C}$ for $24 \mathrm{~h}$, to immobilize Al catalyst via cation exchange. Solid Brønsted acid (BA-MWCNT) was synthesized by contacting excess amount of $1.6 \mathrm{M} \mathrm{HCl}$ aqueous solution with sulfonate-tethered MWCNT after pyrolysis to fully protonate the sulfonate group. 
Table 1. Synthesis conditions of different materials ${ }^{a}$.

\begin{tabular}{|c|c|c|}
\hline Sample Code & Isotopic Labeling & Heating Conditions \\
\hline GT250/10-CNT-GC & $\begin{array}{l}\text { Uniform }{ }^{13} \mathrm{C} \text { : } \\
\text { glucose }\end{array}$ & $\begin{array}{l}\text { Pyrolysis temperature of } 250^{\circ} \mathrm{C} \\
\text { Ramp of } 1{ }^{\circ} \mathrm{C} \text { from room temp to } 120^{\circ} \mathrm{C}, 10^{\circ} \mathrm{C} \\
\text { from } 120^{\circ} \mathrm{C} \text { to } 250^{\circ} \mathrm{C}\end{array}$ \\
\hline GT250/1-GC & $\begin{array}{l}\text { Uniform }{ }^{13} \mathrm{C} \text { : } \\
\text { glucose }\end{array}$ & $\begin{array}{c}\text { Pyrolysis temperature of } 250^{\circ} \mathrm{C} \\
\text { Ramp of } 1^{\circ} \mathrm{C} \text { throughout the temperature range }\end{array}$ \\
\hline GT250/10-CNT-GC/TN & $\begin{array}{l}\text { Uniform }{ }^{13} \mathrm{C} \text { : } \\
\text { glucose } \\
\text { Uniform }{ }^{15} \mathrm{~N} \text { : } \\
\text { taurine }\end{array}$ & $\begin{array}{l}\text { Pyrolysis temperature of } 250^{\circ} \mathrm{C} \\
\text { Ramp of } 1^{\circ} \mathrm{C} \text { from r.t. to } 120^{\circ} \mathrm{C}, 10^{\circ} \mathrm{C} \text { from } 120 \\
{ }^{\circ} \mathrm{C} \text { to } 250^{\circ} \mathrm{C}\end{array}$ \\
\hline GT250/1-GC/TN & $\begin{array}{l}\text { Uniform }{ }^{13} \mathrm{C} \text { : } \\
\text { glucose } \\
\text { Uniform }{ }^{15} \mathrm{~N} \text { : } \\
\text { taurine }\end{array}$ & $\begin{array}{l}\text { Pyrolysis temperature of } 250^{\circ} \mathrm{C} \\
\text { Ramp of } 1^{\circ} \mathrm{C} \text { throughout the temperature range }\end{array}$ \\
\hline GT250/10-CNT-TC & $\begin{array}{l}\text { Uniform }{ }^{13} \mathrm{C} \text { : } \\
\text { taurine }\end{array}$ & $\begin{array}{c}\text { Pyrolysis temperature of } 250^{\circ} \mathrm{C} \\
\text { Ramp of } 1{ }^{\circ} \mathrm{C} \text { from r.t. to } 120^{\circ} \mathrm{C}, 10^{\circ} \mathrm{C} \text { from } 120 \\
{ }^{\circ} \mathrm{C} \text { to } 250^{\circ} \mathrm{C}\end{array}$ \\
\hline GT250/1-TC & $\begin{array}{l}\text { Uniform }{ }^{13} \mathrm{C}: \\
\text { taurine }\end{array}$ & $\begin{array}{c}\text { Pyrolysis temperature of } 250^{\circ} \mathrm{C} \\
\text { Ramp of } 1^{\circ} \mathrm{C} \text { throughout the temperature range }\end{array}$ \\
\hline GT200/1-CNT-TC & $\begin{array}{l}\text { Uniform }{ }^{13} \mathrm{C}: \\
\text { taurine }\end{array}$ & $\begin{array}{l}\text { Pyrolysis temperature of } 200^{\circ} \mathrm{C} \\
\text { Ramp of } 1^{\circ} \mathrm{C} \text { throughout the temperature range }\end{array}$ \\
\hline GT250/1-CNT-TC & $\begin{array}{l}\text { Uniform }{ }^{13} \mathrm{C}: \\
\text { taurine }\end{array}$ & $\begin{array}{l}\text { Pyrolysis temperature of } 250^{\circ} \mathrm{C} \\
\text { Ramp of } 1^{\circ} \mathrm{C} \text { throughout the temperature range }\end{array}$ \\
\hline
\end{tabular}

\subsection{Materials Characterization}

The catalysts were characterized using scanning electron microscopy (SEM, Tokyo, Japan), transmission electron microscopy (TEM, Hillsboro, OR, USA), X-ray photoelectron spectroscopy (XPS, Eden Prairie, MN, USA), $\mathrm{N}_{2}$ adsorption/desorption, and thermogravimetric analysis (TGA, Eden Prairie, MN, USA). TEM images were taken on a Tecnai G2 F20. XPS testing was performed on a Phi 5500 Multitechnique system using an $\mathrm{Al} \mathrm{K} \alpha$ X-ray source. A C1s location of 284.6 was used for peak corrections. TGA experiments were done on a Perkin-Elmer STD 600 under the flow of $50 \mathrm{~mL} / \mathrm{min}$ air, and a temperature ramp of $10^{\circ} \mathrm{C} / \mathrm{min}$, from 50 to $900{ }^{\circ} \mathrm{C}$. $\mathrm{N}_{2}$ adsorption/desorption testing occurred on a Micromeritics ASAP 2020 with the BET method used for surface area calculations (Norcross, GA, USA).

\subsection{Solid-State NMR}

Solid-state magic-angle-spinning NMR studies were carried out on a Bruker Avance 400 spectrometer (Billerica, MA, USA) using $4 \mathrm{~mm}$ and $2.5 \mathrm{~mm}$ magic-angle spinning (MAS) double-resonance probeheads. All ${ }^{13} \mathrm{C}$ experiments were carried out under MAS at $14 \mathrm{kHz}$.

${ }^{13} \mathrm{C}$ was observed at $100 \mathrm{MHz}$. The ${ }^{13} \mathrm{C}$ pulse lengths used were $4 \mu \mathrm{s}$. The samples were prepared according to the synthesis described above and in Table 1. To reduce the conductivity of the MWCNT-containing samples, two additional steps were added to the preparation of some samples: dilution with laponite clay and grinding in a cryomill (Spex SamplePrep). Both of these steps reduce the size of conductive domains within the sample. Conductivity in an ssNMR sample reduces the effectiveness of the radio-frequency pulses through power absorption. The existence of conductivity-induced power loss caused concern that the experiments conducted in the $4 \mathrm{~mm}$ 
probehead could suffer from insufficient ${ }^{1} \mathrm{H}$ decoupling, which could cause the suppression of alkyl ${ }^{13} \mathrm{C}$ peaks in the spectra. In order to confirm that the alkyl peaks were not systematically underrepresented due to poor decoupling, some experiments on the ${ }^{13} \mathrm{C}$-taurine materials were repeated using the $2.5 \mathrm{~mm}$ probehead. A smaller rotor diameter is known to increase effective decoupling power, which reduces possible distortions from incomplete decoupling. The effective decoupling power in the $2.5 \mathrm{~mm}$ probehead was measured by the length of a ${ }^{1} \mathrm{H} 180^{\circ}$ pulse.

Both direct-polarization (DP) and cross-polarization (CP) were used to provide the initial polarization in the MAS ${ }^{13} \mathrm{C}$ NMR experiments. DP experiments were performed with recycle delays $\geq 40 \mathrm{~s}$, and included a Hahn echo. CP experiments used the multiCP pulse sequence [20] in order to obtain quantitative spectra, and also included a Hahn echo.

In order to determine the amount of non-protonated ${ }^{13} \mathrm{C}$ in each sample (aromatized carbon), recoupled dipolar dephasing was applied to both DP and CP experiments. In a recoupled dipolar dephasing experiment, ${ }^{1} \mathrm{H}$ decoupling is removed for a period of time, which causes the signal of protonated ${ }^{13} \mathrm{C}$ nuclei to decrease. The total dipolar dephasing period was made up of two $30 \mu \mathrm{s}$ periods flanking a ${ }^{1} \mathrm{H} 180^{\circ}$ pulse for all recoupled dipolar dephasing experiments.

To further probe the aromatized carbon, we applied ${ }^{13} \mathrm{C}$ REDOR [21] experiments. As the recoupling time in the REDOR experiment increases, ${ }^{13} \mathrm{C}$ nuclei farther and farther from ${ }^{1} \mathrm{Hs}$ are dephased, and their associated signal decreases. Since ${ }^{13} \mathrm{C}$ nuclei closer to ${ }^{1} \mathrm{H}$ nuclei will dephase more quickly, the REDOR experiment is sensitive to distance, and can be particularly useful for probing the size of aromatic domains.

\subsection{Reaction Testing}

Glucose and fructose were tested as model carbohydrate molecules for HMF production with the prepared CNT-based catalysts in a $10 \mathrm{~mL}$ thick-walled glass reactor (Alltech). In a typical experiment, $2.0 \mathrm{~g}$ of the selected organic solvents containing $2 \mathrm{wt} \%$ of glucose or fructose, and one of the solid catalysts, was added to the Alltech reactor, and a triangular stir bar (Fisher Scientific, Hampton, NH, USA) was also added to allow for adequate agitation. The reactor was then sealed with PTFE liner covered caps (Fisher Scientific) and immersed in the oil bath at desired temperatures and stirred at $400 \mathrm{rpm}$. A catalyst recyclability test was performed with fructose as the substrate in the THF/water solvent system. After each run, the organic solvent was decanted, and the catalyst was kept and dried, and washed using sugar-free solvent before fresh solvent containing fructose was added to run the reaction again. In order to test for possible $\mathrm{Al}$ leaching from the catalyst, the decanted supernatant from the fructose reaction was sampled and prepared for ICP analysis.

Reaction product analysis was performed using a Waters 1525 HPLC system equipped with a 2998 PDA UV detector (Milford, MA, USA) at $280 \mathrm{~nm}$, and a 2414 refractive index (RI) detector maintained at $333 \mathrm{~K}$. The aqueous phase samples were analyzed using a PL Hi-Plex H-form carbohydrate column at $353 \mathrm{~K}$ with $5 \mathrm{mM} \mathrm{H}_{2} \mathrm{SO}_{4}$ at a flow rate of $0.6 \mathrm{~mL} \mathrm{~min}^{-1}$ as the mobile phase. The organic phase samples were analyzed using a Zorbax SB-C18 reverse phase column (Agilent, Santa Clara, CA, USA) at $308 \mathrm{~K}$, with a methanol $/ 5 \mathrm{mM} \mathrm{H}_{2} \mathrm{SO}_{4}(8: 2 \mathrm{v} / \mathrm{v})$ binary solvent as the mobile phase at a flow rate of $0.7 \mathrm{~mL} \mathrm{~min}{ }^{-1}$. The conversion/selectivity being used in this manuscript are defined as follows: the conversion of glucose is defined as the moles of glucose reacted, divided by the moles of initial glucose; the selectivity of HMF is defined as the moles of HMF produced divided by the moles of glucose reacted; and the yield of HMF is defined as the glucose conversion multiplied by the HMF selectivity.

\subsection{Inductively Coupled Plasma Mass Spectrometer (ICP-MS) Analysis of Al Leaching}

The aluminum content in each sample was analyzed using an inductively coupled plasma mass spectrometer (ICP-MS) (Bruker Aurora Elite, Billerica, MA, USA). The instrument parameters (Table 1) were optimized for maximum sensitivity with low levels of metal oxide $\left(\mathrm{CeO}^{+} / \mathrm{Ce}^{+} \leq 2 \%\right)$ and doubly charged $\left(\mathrm{Ba}^{2+} / \mathrm{Ba}^{+} \leq 3 \%\right)$ ions. Calibration standards were prepared by serial dilutions of a commercially available aluminum ICP-MS standard (SPEX CertiPrep) with 5\% aqueous hydrochloric 
acid $(\mathrm{HCl})$. The samples were also prepared to have a matrix of $5 \%$ aqueous $\mathrm{HCl}$. The solid MWCNT catalyst samples $(\sim 5 \mathrm{mg})$ were dissolved in concentrated $\mathrm{HCl}$, and then diluted with deionized water to a final $\mathrm{HCl}$ concentration of $5 \%$ (final mass of approximately $10 \mathrm{~g}$ ). The original reaction supernatant samples were diluted 50 - to 100 -fold with $5 \%$ aqueous $\mathrm{HCl}$.

\section{Conclusions}

In summary, the synthesis of sulfonic group-tethered MWCNT solid catalysts has been successfully demonstrated. By manipulating synthesis conditions, catalysts with the desired acid density can be made. Functionalized MWCNTs can be used as a catalyst support to immobilize homogeneous metal cations for various catalytic applications. Solid-state NMR has been shown to be a powerful tool to provide quantitative information about the chemical nature of surface carbon content, in order to optimize those synthesis conditions and observe the changes due to the MWCNT support. The hybrid $\mathrm{Al} / \mathrm{CNT}$ catalysts tested in this work demonstrated good catalyst stability and recyclability for both glucose and fructose dehydration to HMF. More study is required to design a better ligand field on the surface of the MWCNTs, to achieve higher selectivity and less catalyst leaching, and this will be the focus of future research directions.

Author Contributions: H.J. performed the experiments, data collection, and analysis; J.F. analyzed the results; T.W. devised the project. The manuscript was written jointly by all the authors.

Funding: This research was funded by National Natural Science Foundation of China, grant number 21506246.

Conflicts of Interest: The authors declare no conflict of interest.

\section{References}

1. Kobayashi, H.; Fukuoka, A. Synthesis and utilisation of sugar compounds derived from lignocellulosic biomass. Green Chem. 2013, 15, 1740-1763. [CrossRef]

2. Nikolau, B.J.; Perera, M.A.D.; Brachova, L.; Shanks, B. Platform biochemicals for a biorenewable chemical industry. Plant J. 2008, 54, 536-545. [CrossRef] [PubMed]

3. Shanks, B.H. Conversion of Biorenewable Feedstocks: New Challenges in Heterogeneous Catalysis. Ind. Eng. Chem. Res. 2010, 49, 10212-10217. [CrossRef]

4. Ravenelle, R.M.; Schüßler, F.; D'Amico, A.; Danilina, N.; van Bokhoven, J.A.; Lercher, J.A.; Jones, C.W.; Sievers, C. Stability of Zeolites in Hot Liquid Water. J. Phys. Chem. C 2010, 114, 19582-19595. [CrossRef]

5. Tessonnier, J.-P.; Villa, A.; Majoulet, O.; Su, D.S.; Schlögl, R. Defect-Mediated Functionalization of Carbon Nanotubes as a Route to Design Single-Site Basic Heterogeneous Catalysts for Biomass Conversion. Angew. Chem. Int. Ed. 2009, 48, 6543-6546. [CrossRef] [PubMed]

6. Fukuoka, A.; Dhepe, P.L. Sustainable green catalysis by supported metal nanoparticles. Chem. Rec. 2009, 9, 224-235. [CrossRef] [PubMed]

7. Serp, P.; Castillejos, E. Catalysis in Carbon Nanotubes. ChemCatChem 2010, 2, 41-47. [CrossRef]

8. Anderson, J.M.; Johnson, R.L.; Schmidt-Rohr, K.; Shanks, B.H. Solid state NMR study of chemical structure and hydrothermal deactivation of moderate-temperature carbon materials with acidic $\mathrm{SO}_{3} \mathrm{H}$ sites. Carbon 2014, 74, 333-345. [CrossRef]

9. Anderson, J.M.; Johnson, R.L.; Schmidt-Rohr, K.; Shanks, B.H. Hydrothermal degradation of model sulfonic acid compounds: Probing the relative sulfur-carbon bond strength in water. Catal. Commun. 2014, 51, 33-36. [CrossRef]

10. Johnson, R.L.; Anderson, J.M.; Shanks, B.H.; Schmidt-Rohr, K. Simple One-Step Synthesis of Aromatic-Rich Materials with High Concentrations of Hydrothermally Stable Catalytic Sites, Validated by NMR. Chem. Mater. 2014, 26, 5523-5532. [CrossRef]

11. Roman-Leshkov, Y.; Chheda, J.N.; Dumesic, J.A. Phase Modifiers Promote Efficient Production of Hydroxymethylfurfural from Fructose. Science 2006, 312, 1933-1937. [CrossRef] [PubMed]

12. Zhao, Q.; Wang, L.; Zhao, S.; Wang, X.; Wang, S. High selective production of 5-hydroymethylfurfural from fructose by a solid heteropolyacid catalyst. Fuel 2011, 90, 2289-2293. [CrossRef] 
13. Nikolla, E.; Roman-Leshkov, Y.; Moliner, M.; Davis, M.E. “One-Pot” Synthesis of 5-(Hydroxymethyl)furfural from Carbohydrates using Tin-Beta Zeolite. ACS Catal. 2011, 1, 408-410. [CrossRef]

14. Xiong, H.; Wang, T.; Shanks, B.H.; Datye, A.K. Tuning the Location of Niobia/Carbon Composites in a Biphasic Reaction: Dehydration of D-Glucose to 5-Hydroxymethylfurfural. Catal. Lett. 2013, 143, 509-516. [CrossRef]

15. Huang, F.; Su, Y.; Tao, Y.; Sun, W.; Wang, W. Preparation of 5-hydroxymethylfurfural from glucose catalyzed by silica-supported phosphotungstic acid heterogeneous catalyst. Fuel 2018, 226, 417-422. [CrossRef]

16. Zuo, M.; Le, K.; Li, Z.; Jiang, Y.; Zeng, X.; Tang, X.; Sun, Y.; Lin, L. Green process for production of 5-hydroxymethylfurfural from carbohydrates with high purity in deep eutectic solvents. Ind. Crops Prod. 2017, 99, 1-6. [CrossRef]

17. De Souza, R.L.; Yu, H.; Rataboul, F.; Essayem, N. 5-Hydroxymethylfurfural (5-HMF) Production from Hexoses: Limits of Heterogeneous Catalysis in Hydrothermal Conditions and Potential of Concentrated Aqueous Organic Acids as Reactive Solvent System. Challenges 2012, 3, 212-232. [CrossRef]

18. Yang, Y.; Hu, C.-W.; Abu-Omar, M.M. Conversion of carbohydrates and lignocellulosic biomass into 5-hydroxymethylfurfural using $\mathrm{AlCl}_{3} \cdot 6 \mathrm{H}_{2} \mathrm{O}$ catalyst in a biphasic solvent system. Green Chem. 2012, 14, 509-513. [CrossRef]

19. Pagán-Torres, Y.J.; Wang, T.; Gallo, J.M.R.; Shanks, B.H.; Dumesic, J.A. Production of 5-Hydroxymethylfurfural from Glucose Using a Combination of Lewis and Brønsted Acid Catalysts in Water in a Biphasic Reactor with an Alkylphenol Solvent. ACS Catal. 2012, 2, 930-934. [CrossRef]

20. Zhang, Z.; Wang, Q.; Xie, H.; Liu, W.; Zhao, Z.K. Catalytic Conversion of Carbohydrates into 5-Hydroxymethylfurfural by Germanium(IV) Chloride in Ionic Liquids. ChemSusChem 2010, 4, 131-138. [CrossRef] [PubMed]

21. Wang, T.; Pagan-Torres, Y.J.; Combs, E.J.; Dumesic, J.A.; Shanks, B.H. Water-Compatible Lewis Acid-Catalyzed Conversion of Carbohydrates to 5-Hydroxymethylfurfural in a Biphasic Solvent System. Top. Catal. 2012, 55, 657-662. [CrossRef]

22. Johnson, R.L.; Schmidt-Rohr, K. Quantitative solid-state ${ }^{13} \mathrm{C}$ NMR with signal enhancement by multiple cross polarization. J. Magn. Reson. 2014, 239, 44-49. [CrossRef] [PubMed]

(c) 2018 by the authors. Licensee MDPI, Basel, Switzerland. This article is an open access article distributed under the terms and conditions of the Creative Commons Attribution (CC BY) license (http://creativecommons.org/licenses/by/4.0/). 